\title{
Semi-supervised Classification by Probabilistic Relaxation $^{\star}$
}

\author{
Adolfo Martínez-Usó, Filiberto Pla, José Martínez Sotoca, and Henry Anaya-Sánchez \\ Institute of New Imaging Technologies - Dept. of Computer Languages and Systems \\ Universitat Jaume I, 12071 Castellón, Spain
}

\begin{abstract}
In this paper, a semi-supervised approach based on probabilistic relaxation theory is presented. It combines two desirable properties; firstly, a very small number of labelled samples is needed and, secondly, the assignment of labels is consistently performed according to our contextual information constraints. The proposed technique has been successfully applied to pattern recognition problems, obtaining promising preliminary results in database classification and image segmentation. Our methodology has also been evaluated against a recent state-of-the-art algorithm for semi-supervised learning, obtaining generally comparable or better results.
\end{abstract}

Keywords: Semi-supervised, Probabilistic Relaxation, Classification.

\section{Introduction}

Unsupervised learning assumes that all the observations are caused by latent variables that can be somehow modelled. However, there exist many tasks that are generally too specialised to use unsupervised techniques and, at the same time, too time-consuming for an expert if every single case should be solved manually, spending a large amount of time isolating the most interesting parts of the images. These tasks are especially common, for instance in remote sensing or medical imaging applications. Therefore, a process that is able to do this work in an accurate way without too much participation of an expert has become a very demanding task on these fields.

Semi-supervised learning has received an increasing attention for the last years and has been widely extended to many fields [1]. Semi-supervised approaches arise from the idea of using together a large amount of unlabelled data, which is often cheap and easy, and few labelled data, which is hard to obtain since it requires human experts or special devices. The important point here is to manage a better classifier (or clustering result) than from the unlabelled data alone.

Relaxation methods find numerical solutions for a wide range of problems in physics and engineering and, more concretely, probabilistic relaxation has demonstrated to be very useful for pattern recognition [3]. A general framework for the theoretical foundations of relaxation processes can be found in [5]. This general relaxation structure has

\footnotetext{
* This work was supported by the Spanish Ministry of Science and Innovation under the projects Consolider Ingenio 2010 CSD2007-00018, AYA2008-05965-C04-04/ESP, TIN2009-14103C03-01 and by Caixa-Castelló foundation under the projects P1 1B2009-45.
} 
attracted important interest, being often refined in a number of ways by means of $\mathrm{ad}$ hoc or heuristic choices [11].

Relaxation approaches are iterative processes that are used for reducing ambiguities in assigning symbolic labels to a set of nodes (clusters, objects, etc.) which is often known as equilibrating or relaxing a system. Relaxation methods involve contextual information that describes relations between single components [5], defining a neighbourhood in accordance with the properties of the system. The contextual information is generally introduced into the process from our a priori knowledge of the problem. Therefore, these approaches present two interesting features; the use of the context of the problem and the expected good performance to obtain a robust solution [7].

A semi-supervised approach based on probabilistic relaxation is presented in this paper. Using few labelled samples introduced by an expert (contextual information), the proposed method is able to propagate this information to the whole system. Experimental evidences of the robustness of the methodology will be also offered by means of applying the presented technique to several pattern recognition fields such as database classification or image segmentation.

\section{Probabilistic Relaxation Methodology}

A probabilistic relaxation method is an iterative process that assigns consistent labels to a initial set of nodes on the basis of the contextual information, which is also introduced into the model. A node is a point in a graph that represents objects, clusters, regions, etc. whereas the contextual information is generally related to the relationship among those nodes, that is, arcs among the nodes in the graph.

In the proposed approach, an initial Gaussian mixture that models the input data is assumed. Let us consider that each mode of the Gaussian mixture is a node of a fully connected graph. Therefore, let us suppose a set of nodes $\mathcal{N}=\left\{n_{1}, n_{2}, \ldots, n_{N}\right\}$, a set of class labels $\mathcal{L}=\left\{\omega_{1}, \omega_{2}, \ldots, \omega_{L}\right\}$ and a support function $Q^{s}\left(n_{i} \leftarrow \omega_{k}\right)$ representing that the node $n_{i}$ would be labelled with $\omega_{k}$. This support function results from each binary relation with the set $\overline{\mathcal{N}}_{i}$ of neighbouring nodes of $n_{i}$ at the step $s^{t h}$ of the iterative process,

$$
Q^{s+1}\left(n_{i} \leftarrow \omega_{k}\right)=Q^{s}\left(n_{i} \leftarrow \omega_{k}\right)+\frac{1}{\left|\overline{\mathcal{N}}_{i}\right|} \sum_{j \in \overline{\mathcal{N}}_{i}} \mathcal{C}_{i j} P^{s}\left(\omega_{k} \mid n_{j}\right)
$$

where $\mathcal{C}_{i j}$ are the coefficients representing the strength of interaction between nodes $n_{i}$ and $n_{j}$. These coefficients are independent of the estimated posterior probabilities $(P)$ and can be computed ahead of time, remaining constant during the entire process. Coefficients $\mathcal{C}_{i j}$ satisfy that $\sum_{j=1}^{N} \mathcal{C}_{i j}=1$. Our approach defines these coefficients as:

$\mathcal{C}_{i j}=\frac{\mathcal{D}(i, j)}{\sum_{l} \mathcal{D}(i, l)}$, being $\mathcal{D}(i, j)=\exp \left(\frac{c_{i j}}{\kappa}\right)$ where $\kappa=\min \left(c_{m n}\right) \forall m, n \in \mathcal{N}$.

Coefficients $c_{i j}=\frac{\alpha_{i j}}{d(i, j)}$ for nodes $i, j$ represent the relationship between the relative density $\alpha_{i j}$ and the distance $d(i, j)$ between the nodes. Note that $\mathcal{D}(i, j)$ is a potential 
term that acts as a relative measure of potential similarity function. It will be high for the neighbouring node with the best rate for coefficient $c_{i j}$ and very low for the rest of the nodes.

Coefficients $\alpha$ have been worked out using the $d$-dimensional volumes that form each node, being $d$ the dimension of the data. That is, since each node represents a mode of a Gaussian mixture, the radius of each dimension of the ellipse formed by the mode can be derived from the covariance matrix of the mixture mode. In our case, radius is approximated as 3 times the standard deviation. Therefore, if we have $d$ radius for each mode, say $\left\langle r_{1}, r_{2}, \ldots, r_{d}\right\rangle$, the $d$-dimensional ellipsoid volume $\mathcal{V}$ and the density $\rho$ for mode $m$ are calculated as

$$
\mathcal{V}(m)=\frac{\pi^{\frac{d}{2}}}{\Gamma\left(\frac{d}{2}+1\right)} \prod_{i}^{d} r_{i} \quad \rho(m)=\frac{\operatorname{card}(m)}{\mathcal{V}(m)}
$$

where $\Gamma$ is the gamma function and $\operatorname{card}(\cdot)$ provides the number of samples of the mode. In this sense, each sample belongs to the ellipsoid where it is contained, assigning to the nearest ellipse centroid those samples that could belong to several ellipsoids (intersections).

Therefore, using these equations, the coefficient $\alpha$ between the modes $i$ and $j$ is calculated as $\alpha_{i j}=\frac{\rho(i, j)}{\rho(i)+\rho(j)}$, where density $\rho(i, j)$ is worked out considering the possible mode formed by the samples of both modes $i$ and $j$.

The updating formula for calculating the posterior probability $P^{s}\left(\omega_{k} \mid n_{i}\right)$ for label $\omega_{k}$ given the node $n_{i}$ is:

$$
P^{s+1}\left(\omega_{k} \mid n_{i}\right)=\frac{P^{s}\left(\omega_{k} \mid n_{i}\right) Q^{s}\left(n_{i} \leftarrow \omega_{k}\right)}{K}
$$

where $K=\sum_{l=1}^{|\mathcal{L}|} P^{s}\left(\omega_{l} \mid n_{i}\right) Q^{s}\left(n_{i} \leftarrow \omega_{l}\right)$ is a normalising factor.

The system $\left(P^{0}\right)$ is initialised on the basis of the a priori information of the problem statement, also setting $Q^{0}=P^{0}$ in this initialisation. The number of neighbouring nodes $(\overline{\mathcal{N}})$ is set up to the number of classes of the problem.

\subsection{The Algorithm}

The algorithm here presented for a semi-supervised probabilistic relaxation (semi-PR) has three input sources: the number of classes, the labelled data and unlabelled data. The unlabelled data is divided into two subsets for training and test. Multiple initial probability distributions (modes) per class are considered, being the number of modes of each class estimated in the initialisation stage. Thus, semi-PR generates a Gaussian mixture model of the data where each initial mode is a node of the graph used for the probabilistic relaxation described in Sect. 2. Each node of the graph has its initial probabilities according to the contextual information provided by the user and the strength of interaction between nodes is used in the edges of the graph.

Initialising the mixture. The initialisation stage provides the preliminary Gaussian mixture that models the whole dataset as a reduced description of the data. Several 
techniques have been taken into account [910] to learn this initial mixture. However, the more robust behaviour has been found using a vector quantization design [6] for estimating the $N$ centroids that cover the whole dataset and then assigning each sample to the nearest centroid found by the vector quantizer. Thus, the training data is divided into $N$ modes where the mean and covariance of each mode is estimated. Figure 1 shows several examples of this initialisation stage in its second row.

The initialisation stage is finished by a final refinement of the initial mixture, where modes with a high compatibility ( $\alpha$ coefficients) are merged into one. That is, given a pair of modes $i, j$, if the $\alpha_{i j}$ value is higher than certain threshold $\mathcal{T}$, these modes are considered together as a single mode. Figure 1 shows examples of this refinement process in its third row.

It is worth saying that both the initial $N$ and the refinement threshold $\mathcal{T}$ have been set up to values that are safe enough for not creating any mode with instances from different classes. The experimental part of this work describes these values.

Semi-supervised Probabilistic Relaxation Algorithm. The Gaussian modes found in the initialisation stage are used as initial nodes for our proposed Semi-supervised Probabilistic Relaxation Algorithm. The initial probabilities $\left(P^{0}\right)$ for each node are based on the a priori information that stems from our problem, that is, from initial modes found in the initialisation stage and from the supervised samples. These supervised samples allow us to label some of the initial nodes/modes to certain classes. The probabilities of the modes where no a priori information is available are equally distributed for each class before starting the relaxation process.

The objective of the semi-PR algorithm is to iteratively optimise Equation (4) until all the initial nodes would be consistently labelled for each class. For this optimisation, Equation (1) must be also calculated at each iteration, providing the level of agreement for each node and their possible labels. Distance $d(i, j)$ in coefficients $c_{i j}$ is worked out using the Mahalanobis distance between the nodes $n_{i}$ and $n_{j}$.

The process stops when, for each node of the system, one of the class probabilities exceeds $1-\epsilon_{1}$, being $\epsilon_{1} \ll 1$. As the literature suggests [2], if the whole changes in the system do not exceed certain threshold $\epsilon_{2}$, the system is supposed relaxed enough and the process also stops in this case. Note that the process needs to keep iterating until the previous stopping conditions are reached, even so, from the first iterations the propagation of information is channelled through the probabilistic relaxation model. Therefore, parameters $\epsilon_{1}$ and $\epsilon_{2}$ are not critical, although they can probably be considered application-dependent.

\section{Experimental Results}

The experimental part of this work shows the preliminary results obtained by our proposal in several applications. Experiments are divided into the results obtained using several synthetic toy datasets (Sect. 3.1) and the results obtained in colour image segmentation (Sect. 3.2).

Initial mixtures in all the cases have heuristically been set up to 40 modes $(N=40)$, having a refinement threshold $\mathcal{T}=0.5$. In addition, unless otherwise stated, only two 
supervised samples per class are used, which have randomly been selected from the training set. The position of these labelled samples is drawn in the resulting labelled dataset as black-square spots. Samples of the test set are eventually classified performing their maximum class conditional likelihood according to the resulting Gaussian mixture model associated with each class.

\subsection{Results on $2 D$-Datasets}

Figure 1 shows the results obtained on synthetic $2 D$-datasets with two classes. These toy datasets are frequently used in the literature as illustrative examples to show the robustness of a technique. Semi-PR algorithm shows very consistent results with a classification accuracy higher than $95 \%$ in all the cases.

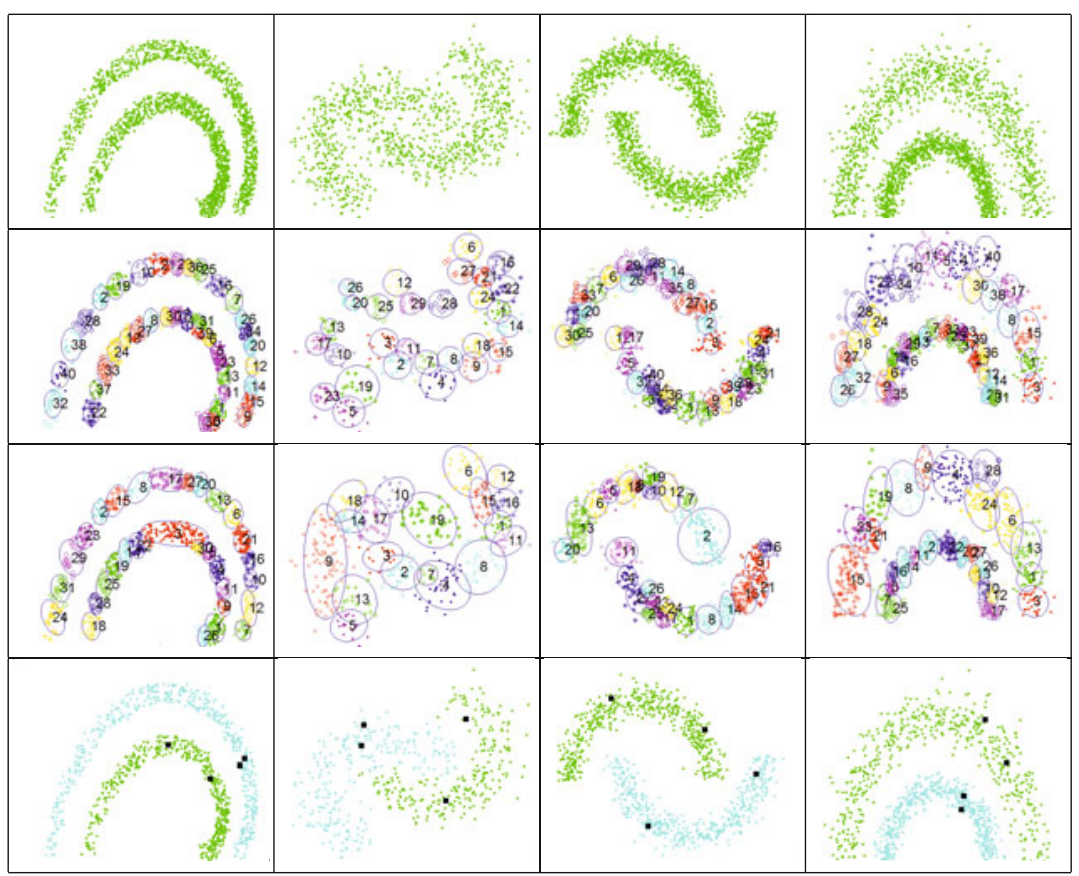

Fig. 1. Experiments on synthetic 2D-datasets with two classes. From top to bottom in rows, input data, preliminary initialisation, initialisation refinement and results of the presented technique.

Figure 2 shows the results obtained on synthetic $2 D$-datasets in a multi-class schema. The first row of the figure shows the input datasets from which, although these datasets are provided unlabelled, is easy to have an idea of which should be the ideal clustering solution. These datasets, let us name DS1, DS2 and DS3 1 from left to right in columns,

\footnotetext{
${ }^{1}$ DS2 and DS3 datasets are available from CLUTO - Software for Clustering High-Dimensional Datasets at http://glaros.dtc.umn. edu/gkhome/cluto/cluto/download.
} 


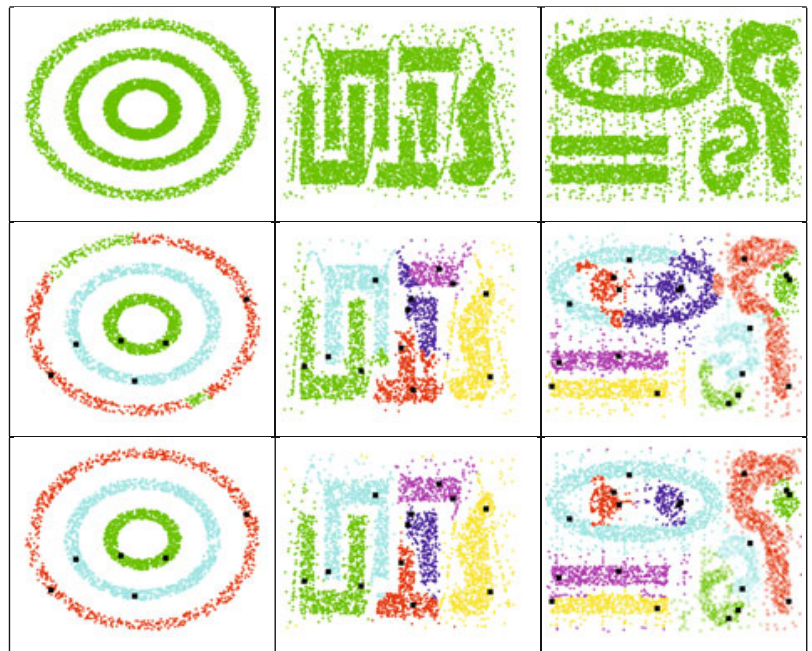

Fig. 2. Experiments on synthetic 2D-datasets multi-class. From top to bottom in rows, input data, results obtained by Semi-LGC and results obtained by the presented technique (Semi-PR).

have been used not only for testing our proposal but for comparing our results with the semi-supervised algorithm proposed by Zhou et al. in [12], hereafter called Semi-LGC. This algorithm is a very well-known work and representative of graph based semisupervised learning approaches. Parameters for the Semi-LGC algorithm have been estimated in a similar way to [8], being the kernel parameter $\sigma$ conveniently defined as the third part of the average distance between each point and its nearest neighbour.

Figure 2 shows in its second row the results obtained by Semi-LGC algorithm whereas the results obtained by our proposal are shown in the third row. As it can be seen, Semi-PR has found more consistent solutions than Semi-LGC for these datasets, solving perfectly DS1 and obtaining an acceptable labelling result for DS2 and DS3. Since Semi-LGC algorithm works in a transductive way, for this experiment no division into training and test sets has been done. The same two supervised samples per class (randomly selected) have been used for Semi-PR and Semi-LGC algorithms.

Finally, it is also important to compare the performance of the two semi-supervised approaches in terms of the classification accuracy for different number of labelled samples. We have used the datasets shown in Figure 1 to this end. Let us name TOY1, TOY2, TOY3 and TOY4 to each column (dataset) of the figure. Again, no division into training and test set has been done and the same supervised samples, randomly selected, per class have been used in both algorithms. Figure 3 shows the classification rate (y-axis) for each semi-supervised method related to the number of labelled samples provided per class (x-axis). Each method has been carried out 10 times in order to reduce the influence of the stochasticity in the experiment.

As it can be seen, both algorithms perform very similar in datasets TOY1, TOY2 and TOY3. However, our proposal clearly outperforms the classification accuracy obtained by Semi-LGC in TOY4. 

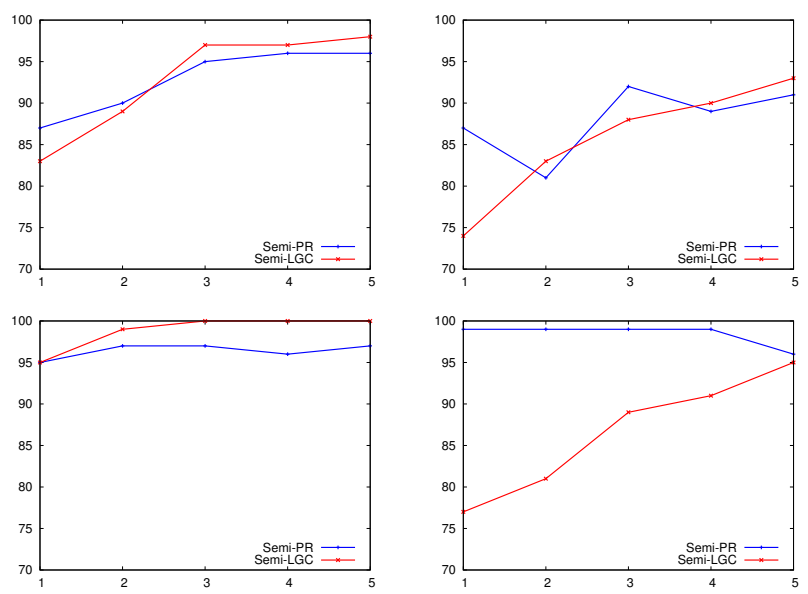

Fig. 3. Performance obtained for Semi-LGC algorithm and our proposal (Semi-PR) related to the number of supervised samples per class used. From left to right and top to bottom, classification accuracy for TOY1, TOY2, TOY 3 and TOY4 datasets.

\subsection{Results on Colour Image Segmentation}

The proposed technique has also been used for segmenting colour images. The total amount of pixels is equally divided into two sets, one for training and another for test. From each image, the number of classes is known and only two labelled pixels per class are provided to the algorithm, which have randomly been taken from the training set. Each sample is a $2 D$-vector representing the chroma of a pixel in the $L^{*} a^{*} b^{*}$ colour space, that is, the $a^{*} b^{*}$ dimensions.

Figure 4 shows the image segmentation results obtained for the well-known images of house and toys. As it can be seen, the segmentation results are quite robust, especially in the case of the house image. The segmentation result for toys image, although acceptable, shows some important mistakes mainly due to the effect of shadows and brightness. Note that there are some isolated pixels that are badly labelled. This is due to the fact that there is no spatial constraint in the process, but some spatial regularisation could be applied as in [4].
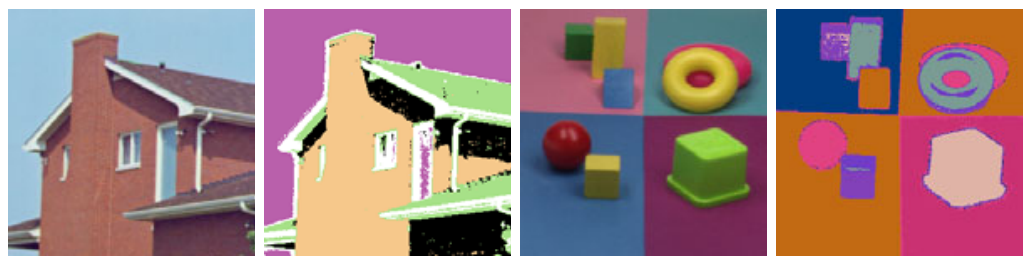

Fig. 4. Image segmentation results. From left to right, source image house (5 classes), segmentation result, source image toys (12 classes) and its segmentation result. 


\section{Conclusions}

The main objective of this work is to develop a robust semi-supervised algorithm using probabilistic relaxation that can be applied to several application problems. Our methodology satisfies the use of few labelled samples and the assignment of labels according to the contextual constraints.

This work supports the increasing attention that the semi-supervised learning is receiving during the last years. The presented approach has demonstrated an effective use of labelled and unlabelled data in experiments including classification in toy data problems and image segmentation. For a classification problem, the accuracy obtained has been comparable to a recent semi-supervised approach from the literature, being capable to reach the highest classification rates with very few labelled samples. For an image segmentation problem, the methodology has demonstrated some promising results in classic images from the literature.

The promising results obtained in terms of the amount of supervised information needed to finish the task allow our proposal to be very suitable for partial annotation. Therefore, in order to extend this work, further actions will include testing the algorithm from a practical point of view in interactive image segmentation or applications in remote sensing or medical imaging. Future work will also include testing the algorithm in other problems $i$ ) with higher dimensionality, ii) in presence of noise and iii) with a higher level of overlapping among the classes.

\section{References}

1. Chapelle, O., Scholkopf, B., Zien, A. (eds.): Semi-supervised Learning. MIT Press (2006)

2. Christmas, W.J.: Structural matching in computer vision using probabilistic reasoning. $\mathrm{PhD}$ thesis, CVSSP, University of Surrey (1995)

3. Christmas, W.J., Kittler, J., Petrou, M.: Structural matching in computer vision using probabilistic relaxation. IEEE Trans. on PAMI 17(8), 749-764 (1995)

4. Diplaros, A., Vlassis, N., Gevers, T.: A spatially constrained generative model and an EM algorithm for image segmentation. IEEE Trans. on Neural Networks 18(3), 798-808 (2007)

5. Faber, P.: A theoretical framework for relaxation processes in pattern recognition: Application to robust nonparametric contour generalization. IEEE Trans. on PAMI 25(8), 1021-1027 (2003)

6. Gersho, A., Gray, R.M.: Vector quantization and signal compression. Kluwer Academic Publishers, Norwell (1992)

7. Haralick, R.M.: Decision making in context. IEEE Trans. on PAMI 5(4), 417-428 (1983)

8. Liu, Q., Liao, X., Li, H., Stack, J.R., Carin, L.: Semisupervised multitask learning. IEEE Trans. on PAMI 31(6), 1074-1086 (2009)

9. Martinez-Uso, A., Pla, F., Sotoca, J.M.: A semi-supervised gaussian mixture model for image segmentation. In: ICPR, pp. 2941-2944 (2010)

10. Shi, T., Belkin, M., Yu, B.: Data spectroscopy: Learning mixture models using eigenspaces of convolution operators. In: ICML 2008, pp. 936-943 (2008)

11. Wang, H., Hancock, E.R.: Probabilistic relaxation using the heat equation. In: ICPR, vol. 2, pp. 666-669 (2006)

12. Zhou, D., Bousquet, O., Lal, T.N., Weston, J., Schölkopf, B.: Learning with local and global consistency. In: Advances in Neural Information Processing Systems, vol. 16, pp. 321-328. MIT Press (2004) 